Original Article

\title{
The Effect of the Abdominal Drawing-in Manoeuvre during Forward Steps
}

\author{
Sachiko Madokoro ${ }^{1)^{*}}$, Hiroichi Miaki ${ }^{1)}$, Toshiaki Yamazaki ${ }^{1)}$ \\ 1) Division of Rehabilitation Science, Department of Health Sciences, Institute of Medical, \\ Pharmaceutical and Health Sciences, Kanazawa University: 5-11-80 Kodatsuno, Kanazawa City, \\ Ishikawa 920-0942, Japan
}

\begin{abstract}
Purpose] A decrease in hip extension has been reported to be a factor in short step width and slow walking speed. Hip motion is related to pelvic and spinal motion, and transversus abdominis (TrA) activation is important for stabilising the pelvis and spine. The abdominal drawing-in manoeuvre (ADIM) can be performed to activate the TrA. The purpose of this study was to examine the influence of the ADIM on forward steps as a gait exercise. [Subjects] The subjects were 20 healthy men (mean age, $20.8 \pm 2.4$ years). [Methods] Thicknesses of the lateral abdominal muscles during forward step posture with and without ADIM were measured using ultrasound, and kinematics of the hip and pelvis were examined using a three-dimensional motion capture system. [Results] Thicknesses of the TrA and internal oblique increased during forward steps with ADIM. In addition, hip extension increased and pelvic rotation and oblique angles decreased during forward step with ADIM. [Conclusion] We believe that ADIM activates the so-called corset muscles, which consequently stabilise the pelvis and spine and increase hip extension. Our results suggest that an ADIM could increase hip extension during gait exercise.

Key words: Abdominal drawing-in manoeuvre, Transversus abdominis, Forward steps
\end{abstract}

(This article was submitted Nov. 25, 2013, and was accepted Jan. 7, 2014)

\section{INTRODUCTION}

Walking is the most common daily activity of locomotion. Gait studies indicate that parameters associated with walking change over time with aging, including decrease of comfortable walking speed, reduction of stride length, increase of step width, prolongation of the stance phase, and shortening of the swing phase. In addition, Murray et al. ${ }^{1)}$ and Kimura et al. ${ }^{2)}$ reported there was a decrease in the amplitude of the total vertical excursion of the head, and slightly less hip and pelvic rotation in older men. Walking speed is correlated with stride length, and it is important for elderly individuals to maintain walking speed in order to enjoy a healthy and active life. Kerrigan et al. ${ }^{3,4)}$ observed healthy elderly subjects and found a reduction in peak hip extension and increased anterior pelvic tilt with decreased walking speed. Hip extension influences the energy cost of walking ${ }^{5}$, therefore, hip extension is an important factor that should be focused on when gait is considered.

During walking, spine and pelvic movements affect hip movement. The pelvic and hip joints also influence each other-a phenomenon known as hip-spine syndrome ${ }^{6,7)}$. For instance, after unilateral total hip arthroplasty, a decrease

*Corresponding author. Sachiko Madokoro (E-mail: shiomoto@mhs.mp.kanazawa-u.ac.jp)

(C2014 The Society of Physical Therapy Science. Published by IPEC Inc. This is an open-access article distributed under the terms of the Creative Commons Attribution Non-Commercial No Derivatives (by-ncnd) License $<$ http://creativecommons.org/licenses/by-nc-nd/3.0/> . in hip extension shows an inverse correlation with the range of motion of pelvic tilt during walking ${ }^{8)}$. Thus, hip extension exercises in the prone position are often performed in rehabilitation. However, in these exercises, patients are often seen performing hip extension with unwanted anterior pelvic tilt and lumbar lordosis. To prevent unwanted lumbar spine and pelvic tilt, stabilisation of the spine is necessary.

Synchronous functioning of the transversus abdominis ( $\operatorname{Tr} A)$ muscle with the multifidus muscle stabilises the spine $^{9,10)}$. Hodges et al. ${ }^{11,12)}$ demonstrated that TrA activation occurs prior to limb movement, independent of movement direction, in asymptomatic adults, but that TrA activation was delayed in patients with low back pain. Furthermore, recruitment of the TrA in individuals with low back pain showed lower electromyographic activity and a smaller increase in TrA thickness during leg tasks than those without low back pain ${ }^{13)}$. Thus, they concluded that a decrease in $\operatorname{Tr}$ A activation was associated with low back pain. Recently, spine stabilisation exercises have become a popular physiotherapeutic treatment for low back pain.

The abdominal drawing-in manoeuvre (ADIM) is commonly used as a basic component of lumbar stabilisation training programs ${ }^{14)}$. Using magnetic resonance imaging, Hides et al. ${ }^{15}$ ) reported that the function of the TrA and thicknesses of the TrA and internal oblique (IO) increased during ADIM. Many other researchers have used ultrasound to demonstrate an increase in TrA thickness during $\mathrm{ADIM}^{16,17)}$. Ultrasound reliably measures thicknesses of the abdominal muscles was reported ${ }^{18)}$. Also, correlation with ultrasound measures thicknesses of the muscles and EMG has been reported ${ }^{19}$. 
Hip extension during ADIM in the prone position improves the relative timing of gluteus maximus activation in relation to the biceps femoris ${ }^{20}$, decreases the electromyographic (EMG) signal amplitude of the erector spinae, increases the EMG signal amplitude of both the gluteus maximus and medial hamstring, and decreases anterior pelvic tilt $^{21)}$. However, few studies have reported on hip extension during ADIM in the standing position or during walking. In healthy elderly subjects, the reduction in peak hip extension and increased anterior pelvic tilt might be related to trunk stabilisation with TrA activation. Thus, walking with ADIM might increase step width. Accordingly, the purpose of the present study was to examine the effects of ADIM during forward steps. Forward steps are performed to practice walking in physical therapy. Yoneda et al. ${ }^{22)}$ measured surface electromyography of the trunk muscles during forward steps, but the TrA was not included.

The hypothesis of the present study was that ADIM during forward steps would increase hip extension and reduce pelvic rotation.

\section{SUBJECTS AND METHODS}

A convenience sample of 20 healthy male volunteers was recruited from a university student population. Their mean age was 20.8 years, their mean weight was $61.5 \pm 5.4 \mathrm{~kg}$, and their mean height was $171.1 \pm 4.1 \mathrm{~cm}$.They had no neuromuscular disease or low back pain at the time of testing. Prior to the study, the principal investigator explained the procedures to all of the subjects. All of the subjects signed an informed consent form, and the study protocol was approved by the Medical Ethics Committee of Kanazawa University.

Measurements were performed in the following postures: in neutral standing, in the forward step posture, and in the forward step posture with ADIM. During neutral standing, subjects were asked to look at a marker set in front of them. During the forward step, subjects stepped forward with their right foot. Prior to stepping, they walked at a selfselected comfortable speed and stride length. After walking, they practiced forward stepping at the same step length as determined while walking comfortably and observing the marker set in front of them.

Subjects practiced ADIM in the standing position, during which we used ultrasound to confirm changes in thickness of the TrA. Ultrasound imaging was performed using a Mylab 25 (Esaote, Indianapolis, IN) in B mode with a 7.5 MHz linear array transducer. The transducer was positioned along the lateral abdominal wall at the anterior axillary line midway between the lowest rib and the iliac crest. The position was determined by visualisation of the fascial planes of the lateral abdominal muscles ${ }^{16,23)}$ and the middle region of the TrA and $\mathrm{IO}^{24)}$. After confirming the fascial planes, we marked each position on the skin to ensure reproducibility of measurement. For the ADIM, subjects were instructed to take in a deep breath, draw their belly button up and in towards the spine as they exhaled, and to hold this position ${ }^{25}$. After they had understood what entailed an ADIM, data were collected in each of the 3 postures using

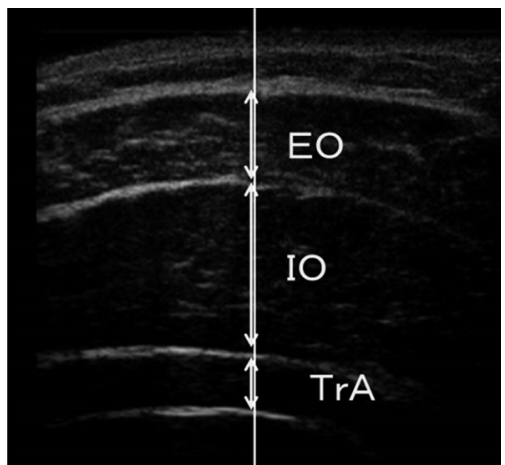

Fig. 1. Ultrasound image The line passing through the middle of the image.

ultrasound and a three-dimensional analysis system.

Ultrasound was used in this study to measure the thicknesses of both sides of the lateral abdominal muscles in each of the 3 postures. Both sides were measured 3 times in each posture. One investigator positioned the transducer and captured all of the images. Each trial was recorded for $10 \mathrm{~s}$.

After muscle thickness measurement using ultrasound, the angles of each hip joint and pelvis segment were measured 3 times using a 3-dimensional motion analysis system (Vicon Motion Systems, Oxford, UK) in each of the 3 postures. The 2 types of step motion were performed at random. Three-dimensional kinematic data were recorded at $250 \mathrm{~Hz}$ using 6 cameras (MT-10; Vicon Motion Systems). Twenty-three reflective markers were placed on anatomic landmarks based on the VICON Plug-in gait and trunk landmarks: C7; Th10; jugular notch; sternum xiphoid; right mid scapula; and both sides of the acromioclavicular joint, anterior superior iliac spine, posterior superior iliac spine, mid-thigh, knee, mid-shank, heel, malleolus, and second metatarsal head. Each trial was recorded from the beginning to the end of the step motion and during stabilisation.

Ultrasound images were selected at the end of inspiration from within the 10 -s recording. Thicknesses of the muscles were measured at the line passing through the middle of the image using the measurement function of MyLab 25 (Fig. 1). Muscle thicknesses were averaged over the 3 measurements of each of the 3 conditions for all subjects.

Motion capture data were processed using VICON Plugin gait to calculate joint angles. Pelvic tilt was measured as the angle in the laboratory sagittal plane between the sagittal pelvic axis and the sagittal laboratory axis. Pelvic rotation was measured as the angle in the pelvic transverse plane between the sagittal axis of the pelvis and the sagittal laboratory axis. The pelvic oblique angle was measured between the plane of the transverse pelvic axis and the plane of the laboratory transverse axis. Hip flexion and extension were measured as the angle between the sagittal thigh axis and the sagittal pelvic axis. Forward step posture was estimated in each trial when the vertical velocity curve of the left heel marker became constant. Hip and pelvic angle data were averaged over the 3 trials of each of the 3 postures for 
Table 1. Muscle thicknesses (in millimetres)

\begin{tabular}{lccc}
\hline Step side & Natural standing & Forward step & $\begin{array}{c}\text { Forward step with } \\
\text { ADIM }\end{array}$ \\
\hline EO & $8.4 \pm 1.4$ & $9.2 \pm 1.7^{*}$ & $9.0 \pm 1.4^{*}$ \\
IO & $11.9 \pm 2.5$ & $13.1 \pm 2.2^{*}$ & $16.9 \pm 2.9^{*} \dagger$ \\
TrA & $5.4 \pm 1.3$ & $6.0 \pm 1.3^{*}$ & $8.2 \pm 1.7^{* \dagger}$ \\
\hline Non-step side & & \\
EO & $8.3 \pm 1.7$ & $7.9 \pm 1.8^{*}$ & $7.9 \pm 1.9$ \\
IO & $12.3 \pm 2.4$ & $11.3 \pm 2.2^{*}$ & $14.6 \pm 2.4^{*} \dagger$ \\
TrA & $5.5 \pm 1.3$ & $5.0 \pm 1.4^{*}$ & $7.8 \pm 2.4^{*} \dagger$ \\
\hline Values are expressed as mean \pm SD & \\
*Significant difference compared with natural standing & \\
†Significant difference compared with forward step & \\
ADIM, abdominal drawing-in manoeuvre; EO, external oblique; IO, internal \\
oblique; TrA, transversus abdominis
\end{tabular}

all subjects.

Muscle thickness and joint angle averages of each individual were expressed as the mean (SD) of all subjects. Comparisons between the neutral standing and step posture and between the two step conditions were made using the paired $t$ test with the Bonferroni correction. Analysis was performed with SPSS Statistics version 19.0.0 (IBM Corporation, Armonk, NY) using $\mathrm{p}=0.05$ as the threshold for significance.

\section{RESULTS}

The intraclass correlation coefficient for reliability was more than 0.90 in neutral standing, more than 0.91 in the forward step posture, and more than 0.85 in the forward step posture with ADIM for all muscles.

For the forward step (right) side, the external oblique (EO) thicknesses in the forward step posture and in the forward step posture with ADIM increased significantly compared with that in neutral standing ( $p<0.01$ and $p<0.05$, respectively). The difference in the forward step side EO thickness between the forward step posture alone and in with the forward step posture with ADIM was not significantly different. Similarly, the thicknesses of the $\operatorname{Tr} A$ in the forward step posture and the forward step posture with ADIM were significantly increased compared with that during neutral standing ( $\mathrm{p}<0.05$ and $\mathrm{p}<0.01$, respectively). Additionally, TrA thickness in the forward step posture with ADIM was significantly increased compared with that in the forward step posture alone $(\mathrm{p}<0.01)$. The IO results were similar to those of the TrA.

For the nonstep (left) side, the EO thickness in the forward step posture was significantly smaller than that in neutral standing $(\mathrm{p}<0.05)$, but there was no significant difference between neutral standing and the forward step posture with ADIM. Moreover, the nonstep side EO thickness during the forward step posture alone was not significantly different from that in the forward step posture with ADIM. The TrA thickness in the forward step posture was significantly smaller than that during neutral standing $(\mathrm{p}<0.01)$. In addition, the thickness of the TrA in the forward step posture with ADIM was significantly increased compared with that in neutral standing $(\mathrm{p}<0.01)$. Additionally, the thickness of the TrA in the forward step posture was significantly different from that in the forward step posture with ADIM $(p<0.01)$. The IO results were similar to those of the TrA (Table 1).

For the forward step side, hip flexion angles in the forward step posture and in the forward step posture with ADIM were significantly larger than that during neutral standing $(\mathrm{p}<0.01)$. The hip flexion angle of the step side during the forward step posture alone was not significantly different from that in the forward step posture with ADIM. For the non-step side, the hip extension angles in the forward step posture and in the forward step posture with ADIM were significantly larger than that in neutral standing $(\mathrm{p}<0.01)$. Additionally, the hip extension angle in the forward step posture with ADIM was significantly larger than that in the forward step posture alone $(\mathrm{p}<0.05)$.

There were no significant differences in pelvic tilt. The pelvic oblique angle was significantly inclined on the left side in the forward step posture and the forward step posture with ADIM compared with that in neutral standing ( $p$ $<0.01$ ). No significant difference was seen between the forward step posture alone and that with ADIM. Pelvic rotation in the forward step posture and in the forward step posture with ADIM was to the left of that in neutral standing ( $p$ $<0.01$ ). Additionally, pelvic left rotation in the forward step with ADIM was significantly lower than that in the forward step posture alone $(\mathrm{p}<0.05)$ (Table 2$)$.

\section{DISCUSSION}

The results of the present study show that the muscles on the step side in the forward step posture were significantly thicker than in neutral standing. Correspondingly, the thicknesses of the muscles on the non-step side decreased. The relative increase observed in the IO on the step side was similar to that determined by Yoneda et al. ${ }^{22)}$, who reported that relative IO muscle activity on the step side increased in forward stepping, as measured by surface electromyography. In addition, in the present study, the thicknesses of the EO and TrA on the step side increased in the forward step posture. Hu et al. ${ }^{26)}$ showed that all lateral abdominal 
Table 2. Hip and pelvic angles

\begin{tabular}{lccc}
\hline Angle (in degrees) & Natural standing & Forward step & $\begin{array}{c}\text { Forward step with } \\
\text { ADIM }\end{array}$ \\
\hline Right hip flexion & $3.3 \pm 5.2$ & $10.1 \pm 6.2^{*}$ & $10.2 \pm 6.8^{*}$ \\
Left hip extension & $1.5 \pm 5.1$ & $-7.3 \pm 7.3^{*}$ & $-10.6 \pm 6.8^{* \dagger}$ \\
Pelvic tilt & $11.5 \pm 4.2$ & $11.8 \pm 3.3$ & $11.3 \pm 4.7$ \\
Pelvic oblique & $1.2 \pm 1.8$ & $-2.0 \pm 2.0^{*}$ & $-1.8 \pm 2.3^{*}$ \\
Pelvic rotation & $0.6 \pm 2.6$ & $-4.8 \pm 3.9^{*}$ & $-3.7 \pm 3.9^{*} \dagger$ \\
\hline
\end{tabular}

Values are expressed as mean \pm SD

*Significant difference compared with natural standing

$\dagger$ Significant difference compared with forward step

ADIM, abdominal drawing-in manoeuvre

muscles on the stepping side are active during the stride cycle of gait, which indicates stabilisation, and our results are similar to those reported by their study $\mathrm{Hu}$ et al. also reported that the TrA on the swing side and the EO on the opposite side are active during the swing cycle. Thus, the TrA on the contralateral swing side may be active; however, this is not clear.

The 3D motion analysis showed that in the forward step posture, pelvic tilt did not occur, but that lateral tilt and left rotation occurred. We found that the motion of the forward step shifts the weight to the step side, and that the lower limb of the non-step side moves such that only the tip of the toe remains on the ground. The results of a preliminary experiment showed that lateral abdominal muscle thicknesses and the pelvic angle in one-leg standing did not change from those of neutral standing. Urquhart et al. ${ }^{27)}$ reported there was greater activity in the lower and middle regions of the contralateral TrA and in the lower region of the contralateral IO during left rotation, and that the upper regions of the ipsilateral TrA and EO are predominantly active during right rotation. In this study, we measured the thicknesses of the middle regions of the TrA and IO. Both thicknesses increased along with pelvic rotation to the opposite side. It is not clear whether the difference between sitting and standing might be related to the activity of the EO. Further examination is necessary to clarify the relationship between the change in pelvic angle and lateral abdominal muscle thickness.

The thicknesses of the IO and TrA on both sides were increased in the forward step posture with ADIM compared with those of neutral standing. Based on this result, we believe that ADIM causes the IO and TrA activation on both sides.

The results of the kinematic analysis show that ADIM increased hip extension in the forward step posture, confirming the hypothesis of our present study. Konz et al. ${ }^{28)}$ investigated walking with and without spinal restriction using a fiberglass body jacket at 5 different speeds. They reported that peak-to-peak pelvic obliquity and rotation were significantly reduced across all walking speeds with spinal restriction, while peak-to-peak hip flexion-extension significantly increased at only the slow and very slow speeds. In our present study, hip extension in the forward step posture with ADIM might have been increased by the action of ADIM replicating that of a body jacket, restricting spine and pelvic motion. Moreover, Konz et al. ${ }^{28)}$ reported that the peak-to-peak pelvic tilt was significantly reduced only at the fastest speed. In our study, pelvic tilt was not different between the forward step with and without ADIM. This is probably because the forward step in the present study was a single-step motion, and because it was similar at a slow speed. Hu et al. ${ }^{29)}$ investigated muscle activity during treadmill gait with and without a pelvic belt, and found that the activities of the transverse and oblique muscles of the abdominal wall, iliacus, erector spinae, and biceps femoris were reduced by a pelvic belt, while that of the gluteus maximus was increased. They suggested that the pelvic belt substituted for pelvic-stabilizing activity. Chance-Larsen et al. ${ }^{20)}$ reported that prone hip extension with ADIM possibly improves the relative timing of gluteus maximus activation in relation to the biceps femoris. The increase in hip extension seen in our study may have been related to the activity of the gluteus maximus, but we did not measure gluteus maximus activation. Further investigation of this muscle's activity is necessary.

We studied young, healthy, male volunteers who could perform ADIM with accuracy; thus, it is not clear whether comparable results would be obtained in other age groups and females. Moreover, we measured pelvic and hip angles but did not establish whether step width increased. It will be necessary to measure step width and to confirm the influence of the change in hip extension in a future study. Moreover, we did not measure the activity of the back muscles, which are considered to influence stability of the trunk and pelvis; therefore, we need to investigate back muscle activity in the future. In addition, muscle activity of the lower limbs should also be evaluated in future studies.

\section{ACKNOWLEDGEMENTS}

The authors express their sincere thanks to the subjects and acknowledge Dr. Takao Nakagawa's help with the preparation of this manuscript.

\section{REFERENCES}

1) Murray MP, Kory RC, Clarkson BH: Walking patterns in healthy old men J Gerontol, 1969, 24: 169-178. [Medline] [CrossRef]

2) Kimura $T$, Kobayashi H, Nakayama E, et al.: Effects of aging on gait patterns in the healthy elderly. Anthropol Sci, 2007, 115: 67-72. [CrossRef] 
3) Kerrigan DC, Lee LW, Collins JJ, et al.: Reduced hip extension during walking: healthy elderly and fallers versus young adults. Arch Phys Med Rehabil, 2001, 82: 26-30. [Medline] [CrossRef]

4) Kerrigan DC, Todd MK, Della Croce U, et al.: Biomechanical gait alterations independent of speed in the healthy elderly: evidence for specific limiting impairments. Arch Phys Med Rehabil, 1998, 79: 317-322. [Medline] [CrossRef]

5) Wert DM, Brach J, Perera S, et al.: Gait biomechanics, spatial and temporal characteristics, and the energy cost of walking in older adults with impaired mobility. Phys Ther, 2010, 90: 977-985. [Medline] [CrossRef]

6) Offierski CM, MacNab I: Hip-spine syndrome. Spine, 1983, 8: 316-321. [Medline] [CrossRef]

7) Matsuyama $Y$, Hasegawa $Y$, Yoshihara H, et al.: Hip-spine syndrome: tota sagittal alignment of the spine and clinical symptoms in patients with bilateral congenital hip dislocation. Spine, 2004, 29: 2432-2437. [Medline] [CrossRef]

8) Miki H, Sugano N, Hagio K, et al.: Recovery of walking speed and symmetrical movement of the pelvis and lower extremity joints after unilatera THA. J Biomech, 2004, 37: 443-455. [Medline] [CrossRef]

9) Richardson CA, Jull GA: Muscle control-pain control. What exercises would you prescribe? Man Ther, 1995, 1: 2-10. [Medline] [CrossRef]

10) Richardson CA, Jull GA, Hodges PW, et al.: Therapeutic Exercises for Spinal Segmental Stabilization in Low Back Pain: Scientific Basis and Clinical Approach. New York: Churchill Livingstone, 1999.

11) Hodges PW, Richardson CA: Inefficient muscular stabilization of the lumbar spine associated with low back pain. A motor control evaluation of transversus abdominis. Spine, 1996, 21: 2640-2650. [Medline] [CrossRef]

12) Hodges PW, Richardson CA: Contraction of the abdominal muscles associated with movement of the lower limb. Phys Ther, 1997, 77: 132-142, discussion 142-144. [Medline]

13) Ferreira PH, Ferreira ML, Hodges PW: Changes in recruitment of the abdominal muscles in people with low back pain: ultrasound measurement of muscle activity. Spine, 2004, 29: 2560-2566. [Medline] [CrossRef]

14) Teyhen DS, Miltenberger CE, Deiters HM, et al.: The use of ultrasound imaging of the abdominal drawing-in maneuver in subjects with low back pain. J Orthop Sports Phys Ther, 2005, 35: 346-355. [Medline] [CrossRef]

15) Hides J, Wilson S, Stanton W, et al.: An MRI investigation into the function of the transversus abdominis muscle during "drawing-in" of the abdominal wall. Spine, 2006, 31: E175-E178. [Medline] [CrossRef]

16) Teyhen DS, Gill NW, Whittaker JL, et al.: Rehabilitative ultrasound imaging of the abdominal muscles. J Orthop Sports Phys Ther, 2007, 37 450-466. [Medline] [CrossRef]

17) Jhu JL, Chai HM, Jan MH, et al.: Reliability and relationship between 2 measurements of transversus abdominis dimension taken during an abdominal drawing-in maneuver using a novel approach of ultrasound imaging. J Orthop Sports Phys Ther, 2010, 40: 826-832. [Medline] [CrossRef]

18) Hodges PW, Pengel LH, Herbert RD, et al.: Measurement of muscle contraction with ultrasound imaging. Muscle Nerve, 2003, 27: 682-692. [Medline] [CrossRef]

19) McMeeken JM, Beith ID, Newham DJ, et al.: The relationship between EMG and change in thickness of transversus abdominis. Clin Biomech (Bristol, Avon), 2004, 19: 337-342. [Medline] [CrossRef]

20) Chance-Larsen K, Littlewood C, Garth A: Prone hip extension with lower abdominal hollowing improves the relative timing of gluteus maximus activation in relation to biceps femoris. Man Ther, 2010, 15: 61-65. [Medline] [CrossRef]

21) Oh JS, Cynn HS, Won JH, et al.: Effects of performing an abdomina drawing-in maneuver during prone hip extension exercises on hip and back extensor muscle activity and amount of anterior pelvic tilt. J Orthop Sports Phys Ther, 2007, 37: 320-324. [Medline] [CrossRef]

22) Yoneda H, Suzuki T: Muscle activity of the trunk muscles during a back and forward steps of the right leg in standing of healthy persons: examination of a forward step and a back step. Kansai Physiother, 2007, 7: 85-90 (in Japanese).

23) Rankin G, Stokes M, Newham DJ: Abdominal muscle size and symmetry in normal subjects. Muscle Nerve, 2006, 34: 320-326. [Medline] [CrossRef]

24) Urquhart DM, Barker PJ, Hodges PW, et al.: Regional morphology of the transversus abdominis and obliquus internus and externus abdominis muscles. Clin Biomech (Bristol, Avon), 2005, 20: 233-241. [Medline] [CrossRef]

25) McGalliard MK, Dedrick GS, Brismée JM, et al.: Changes in transversus abdominis thickness with use of the abdominal drawing-in maneuver during a functional task. PM R, 2010, 2: 187-194, quiz 226. [Medline] [CrossRef]

26) $\mathrm{Hu} \mathrm{H}$, Meijer OG, Hodges PW, et al.: Control of the lateral abdominal muscles during walking. Hum Mov Sci, 2012, 31: 880-896. [Medline] [CrossRef]

27) Urquhart DM, Hodges PW: Differential activity of regions of transversus abdominis during trunk rotation. Eur Spine J, 2005, 14: 393-400. [Medline] [CrossRef]

28) Konz R, Fatone S, Gard S: Effect of restricted spinal motion on gait. J Rehabil Res Dev, 2006, 43: 161-170. [Medline] [CrossRef]

29) $\mathrm{Hu} \mathrm{H}$, Meijer OG, van Dieën JH, et al.: Muscle activity during the active straight leg raise (ASLR), and the effects of a pelvic belt on the ASLR and on treadmill walking. J Biomech, 2010, 43: 532-539. [Medline] [CrossRef] 